\title{
A Smartphone-based Post-Disaster Management Mechanism Using WiFi Tethering
}

\author{
Sayan Kumar Ray ${ }^{1}$, Roopak Sinha ${ }^{2}$, Swapan Kumar Ray ${ }^{3}$ \\ ${ }^{1}$ Faculty of Business and Information Technology, Manukau Institute of Technology, Auckland, New Zealand \\ ${ }^{2}$ School of Computer and Mathematical Sciences, Auckland University of Technology, Auckland, New Zealand \\ ${ }^{3}$ Dept of Computer Science and Engineering, Jadavpur University, Kolkata, India \\ sayan.ray@manukau.ac.nz,roopak.sinha@aut.ac.nz,skray@ieee.org
}

\begin{abstract}
Natural disasters often cause the breakdown of the power grid in the affected areas hampering telecommunication services. In the absence of electrical power undamaged cellular base stations switch to battery backup to sustain communication. However, owing to the sudden substantial increase in voice and data traffic, base stations become over-congested fast and battery backups quickly get exhausted. We propose a smartphone-based post-disaster management mechanism for managing traffic in the affected areas using the concept of WiFi tethering. Smartphones in the affected areas may turn themselves into temporary WiFi hotspots to provide internet connectivity and important communication abilities to nearby WiFi-enabled user devices. The hotspots can self-assess the number of new connections they can serve based on their leftover battery energy. Client devices, approaching the affected areas, can also self-select the most suitable hotspot to connect to depending on their proximity and nature of motion with respect to individual hotspots. Long Term Evolution-Advanced (LTE-A) underlying networks are considered for this work.
\end{abstract}

Keywords-natural disaster, WiFi tethering, smartphones, eNB, LTE-A, leftover energy, direction of motion, network congestion

\section{INTRODUCTION}

Natural disaster incidents, like earthquakes and hurricanes, often cause the breakdown of the electrical power grid leaving the cellular base stations in the affected regions to run on battery backups. The battery backups generally last for 4-8 hours under normal network traffic condition [1-2], but immediately after a disaster incident, owing to the sudden and huge increase in network traffic/calls in the affected areas, the backups do not generally last over few minutes. This leads to a complete and sudden collapse of the whole wireless network infrastructure. Immediately after the Japan tsunami and earthquake in 2011, voice calls in the NTT DoCoMo's mobile communication network increased to almost 50 to 60 times the normal volume of traffic and carriers were forced to restrict voice traffic by as much as 70 to $95 \%$. Moreover, a 4 to 9 times increase in fixed line communication traffic forced carriers to also restrict phone traffic by 80 to $90 \%$ [3]. Packetbased transmissions such as text messages were less affected, but excessive congestion in the base stations and other servers caused significant delays with the delivery of text messages.

A network outage occurs owing to power outage and the consequent traffic congestion. Network traffic congestion of the base stations in an LTE-A network (called LTE Evolved Node B or eNB) in the affected areas can be caused by (a) the sudden substantial increase in voice calls to and from people stuck in the affected areas; and (b) the addition of new communication traffic generated by the rescue and relief team members and other people arriving for rendering help. Addition of new communication to the already over-congested and ailing eNBs only makes the situation worse forcing the eNBs to die down faster because battery backups drain rapidly. It is desirable to maintain all or at least a considerable connections with some kind of communication ability without adding more pressure to the congested eNBs in terms of available bandwidth for communication. In the context of such situations, we propose here a smartphone-based post-disaster management mechanism in the disaster affected areas using the concept of WiFi tethering. When using WiFi tethering, a wireless device (a smartphone in this case) can operate either as a mobile WiFi hotspot or as a client [4]. If a mobile device operates as a hotspot then it enables other client devices, within its communication range, to communicate to each other and share the internet connectivity through it. Thus, depending on its leftover energy (LoE) in the battery, WiFi tetheringenabled smartphones belonging to users stuck in the affected areas can form small wireless local area networks to provide internet connectivity and important communication to other WiFi-enabled devices (e.g., other smartphones/cell phones). From here onwards, the smartphone hotspots and clients will be referred to as User Equipment (UE) hotspots and UE clients.

In the proposed post-disaster management mechanism, a smartphone stuck in the disaster affected region under the coverage of an over-congested eNB can turn itself to a temporary WiFi hotspot, through WiFi tethering, depending on its leftover battery energy, which it is able to self-assess. Also, from its LoE, the UE hotspot can itself roughly understand how many new UE client connections it may support before the LoE of its own battery drops down to a certain pre-defined threshold. The UE hotspots broadcast this information periodically for new UE clients approaching the disaster affected areas. A UE client can choose to connect to one of the available WiFi mobile hotspots based on (a) its 
proximity to hotspots coupled with the self-prediction of the magnitude and direction of its motion with respect to the individual hotspots, and (b) the number of available connections advertised by each hotspot. The UE clients assign scores to each of these parameters to individual hotspots and then select (i.e., connect to) the one with the highest weighted average score (WAS).

The aim of the proposed mechanism is twofold. On one hand, the WiFi tethering hotspots can relieve the over congested eNBs (in the affected areas) which are already running low on battery backup power from getting more congested. The proposed scheme can help limit the number of new clients connecting to available eNBs, consequently reducing per-eNB load and allowing eNBs to remain alive for longer. On the other hand, clustered UE clients can sustain important communication for longer through their temporary host UE hotspots.

The rest of this paper is organized as follows. Section II provides a brief review of related works. The proposed disaster management scheme is discussed in Section III. The details of the hotspot selection mechanism are discussed in Section IV. Section V provides an illustrative example and concluding remarks appear in Section VI.

\section{RELATED WORK}

Traffic congestion in the eNBs in the disaster affected areas cause significant damage to the overall network performance. In network congestion the connectivity ratio is decreased by exceeding the communications service processing capacity [5]. Moreover, owing to outage in electrical power, undamaged eNBs in affected regions switch to battery backup that dies down very quickly. Although extremely necessary, research related to setting up robust mobile network infrastructures immune to natural disasters, dynamic traffic offloading and congestion control of affected networks, prioritizing and processing high importance traffic, increasing the backup power of eNBs, setting up post-disaster smart location tracking systems etc., to help out the rescue and relief teams, are not very common as of yet [6].

In recent times, few counter mechanisms have been proposed that either aim at increasing the backup power in base stations/eNBs and/or setting up disaster management/resilient networks. Few mechanisms include the use of fuel cells and hydrogen back up power supply and providing base stations with powerful generators that are able to sustain longer [7-8]. Shibata et. al. have proposed a robust disaster management network constituting self-powered fixed base stations, cognitive mobile base stations and wireless balloon stations [9]. A multi-hop wireless access network for disaster response that can be established on-the-fly, with the purpose of extending internet connectivity from surviving WiFi access points to the mobile phones of the disaster affected people, is proposed in [10]. Users in the affected areas may be able to access free calls and text messaging service through the use of EmergeNet, a portable, rapidly deployable, small scale cellular network [11]. Ad hoc networks also play a role in providing communication in natural disaster situations [12-13]. Research works related to effective congestion control in disaster affected areas and traffic prioritizing mechanisms mostly focus on using virtualization techniques [5], [14].

Barring few stray efforts [4], research initiatives on utilizing the concept of $\mathrm{WiFi}$ tethering in natural disaster management is highly lacking. The scheme presented in [4] assumes a complete breakdown of cellular infrastructure and uses WiFi tethering based ad hoc networks as the only means of communication. On the contrary our scheme aims to relieve congestion on base stations by limiting the number of devices connected to base stations while allowing other devices to maintain communications using WiFi tethering. With the proliferation of $\mathrm{WiFi}$ tethering-enabled smartphones these days, we believe it is possible to utilize this technology in providing important and emergency network communication in disaster affected areas with virtually no setup cost.

\section{Proposed Post-Disaster ManAgement Scheme}

In the proposed mechanism a smartphone connected to an over congested eNB, also running under very little LoE in the battery, understands the occurrence of the unnatural incident and checks its leftover battery energy to self-assess its capability of serving as a temporary WiFi hotspot. Provided it has adequate LoE in the battery, the smartphone turns itself into a temporary $\mathrm{WiFi}$ hotspot and provides internet connectivity to other WiFi-enabled clients (e.g., other user devices) in the affected area. Also, depending on its individual LoE in the battery, each hotspot roughly assesses the number of new connections it can serve before its LoE drops down to a certain threshold value, which it conserves for meeting its own needs. The hotspot advertises its presence, along with the required association credentials and the number of connections it can serve periodically. New users with WiFi-enabled smartphones/devices approaching the affected areas may scan the nearby wireless channels and themselves choose among multiple such available WiFi mobile hotspots to connect to (for internet connectivity) based on two independent criteria. The criteria are: (a) The nature of motion (NoM) of the new users relative to each hotspot, which is self-assessed by each new user by its current proximity relative to each hotspot as well as by the direction and the magnitude of its motion relative to each hotspot, and (b) the number of available connections advertised by each hotspot. Criterion (a) allows new users to enjoy the maximum signal strength while criterion (b) would assure new users about receiving the best quality of service for the longest duration. A client UE assigns scores to each of the nearby hotspots against these two parameters and finally connects to the one receiving the highest score (WAS). Once connected, client UEs carry out important communication as required until the LoEs of the host hotspots drop below the pre-defined threshold beyond which the WiFi tethering terminates.

The mechanism proposed in this paper discusses three key research questions in this context: (a) how can a smartphone UE know about the occurrence of a natural disaster situation before deciding to enable WiFi tethering?, (b) how can a 
mobile hotspot self-assess, at any point of time, the number of connections it can serve?, and (c) how can a new UE client self-assess its NoM with respect to the advertised mobile hotspots? Here, we briefly discuss about these points. Immediately after a disaster situation, eNBs in the affected areas face a significant surge in network traffic leading to congestion in the ailing network. We assume that the congested eNBs, running under lowered battery backups can observe increase in congestion and broadcast the increasing traffic load (spike) periodically. A mechanism of an eNB selfassessing the occurrence of traffic congestion in natural disaster situation is proposed in [15]. With the advent of selforganizing networks (SON), where eNBs are able to track and exchange their load information with each other over the backbone network, such an assumption is not unrealistic [1617]. Moreover, for energy saving purpose, it is also expected that in future cellular networks, adjacent eNBs can cooperate more with each other through the exchange of power-related information over the backbone network [18]. From these periodic load information-related broadcasts, the user UEs under the coverage areas of affected eNBs can learn about the occurrence of an unnatural incident.

A smartphone hotspot can, at any time, roughly determine the number of new connections it can serve based on its LoE, the existing number of connections it is presently serving and its pre-chosen energy threshold. The concept of LoE is discussed in detail in Section IV. For new UE clients approaching the disaster areas, identifying the most suitable hotspot to connect to is a twofold approach. First, for each hotspot nearby, an UE client periodically measures the current relative distance between itself and the hotspot. Simultaneously, using the same set of periodic measurement results, the UE client self-estimates its current NoM with respect to each hotspot. Specifically, the UE client determines whether its relative direction of motion (DoM) is progressive or regressive and also estimates the magnitude of the progression or regression. Based on these measurements the UE client assigns individual NoM scores $\left(\mathrm{S}_{\mathrm{NoM}}\right)$ to each hotspot in the manner explained in Section IV. Secondly, based on the number of new connections each hotspot can presently serve, the UE client assigns connection scores $\left(\mathrm{S}_{\mathrm{CON}}\right)$ to each of them. Finally, by combining these two scores, namely, $\mathrm{S}_{\mathrm{NoM}}$ and $\mathrm{S}_{\mathrm{CON}}$, the client chooses for connecting to the hotspot with the highest overall WAS. Detailed discussions on these points are provided in Section IV.

\section{Selecting the Most Appropriate Hotspot}

When new UE clients move to the disaster affected areas and learn about the disaster from the broadcasts of the temporary WiFi hotspots, they periodically scan the wireless channels to look for advertisements from the different potential hotspots to connect to. Considering that there are multiple hotspot options available, to realize a reliable connection, an UE client ideally seeks to select as its target the hotspot (a) that is presently nearest to it, (b) towards which it is presently approaching the fastest, and (c) which has the maximum LoE to serve new connections. Clearly, all these criteria would help the UE client to choose the hotspot which can provide the most satisfactory quality of service.

\section{A. Proximity and DoM-based Scoring}

An UE client employs a distance estimation-based look ahead scheme to select the hotspot that best matches with its overall NoM relative to all the nearby hotspots. This is done by first estimating its proximity to each hotspot from its present location and by determining its magnitude and DoM relative to the different hotspots. The concept of distance estimation-based look ahead was initially proposed in Ray et. al. [19] and later used in context to Mobile WiMAX handover [20]. In this proposal, a modified version of the distance estimation-based look ahead technique along with a novel score assigning mechanism is used. The score is assigned by an UE client to each nearby mobile hotspot based on the proximity (relative distance) and the magnitude-cum-DoM (MDoM) of the relative motion.

\section{Assignment of proximity-based scores:}

Distance estimation is based on the following key idea: any station, fixed or mobile, can, at any time, roughly estimate its present distance from any other station, fixed or mobile, by measuring the Received Signal Strength (RSS), which is the strength of the signal received from the other station and using an appropriate path loss relationship [19-20]. Thus, a new UE client can roughly estimate its proximity (nearness) to each of the nearby hotspots (these can be mobile as well) by measuring the respective RSSs of the hotspots. Here it is assumed that all hotspots transmit their advertisements at the same power. Now, we assume that (a) the client scans the RSS values for each available hotspot multiple times at regular intervals and calculates the corresponding set of distances and (b) the UE client's motion remains "broadly linear" over a certain time frame. Depending on the results of distance estimation obtained from multiple scannings, a UE client assigns individual proximity-based score $S_{\text {PRO }}$ to each hotspot. SPRO to any particular hotspot is assigned by a UE client as the difference between its maximum distance to any hotspot computed over all the scanning cycles and its most recently computed distance from this particular hotspot. Obviously, this will ensure that the UE client will assign, as is to be desired, the highest $\mathrm{S}_{\mathrm{PRO}}$ to the nearest hotspot seen by it during the latest or most recent scanning.

\section{Assignment of Magnitude-cum-DoM (MDoM)-based scores:}

Based on the sequence of distance estimation values $\left\{\mathrm{d}_{\mathrm{i}}\right\}, \mathrm{i}$ $=1,2,3, \ldots, n$, calculated at the regular intervals, $\left\{t_{1}, t_{2}, t_{3}, \ldots\right.$, $\left.t_{n}\right\}$, the UE client performs a look ahead to self-track its magnitude-cum-DoM via simple computation of its relative velocity with respect to each of the hotspots. This, on one hand, enables the UE client to understand from the DoM whether its movement direction with respect to each hotspot is progressive or regressive, i.e., whether it's moving towards or away from a particular hotspot; on the other hand, it also enables the UE client to identify, from the magnitude of the motion, the hotspot it is most likely to come closest to. This 
look ahead is important because, in spite of a linear motion, there are possibilities that the UE client steers away from the nearest hotspot at the last moment. The technique of look ahead is explained as follows. If the distance $\mathrm{d}$ between a UE client and a hotspot is estimated as $\mathrm{d}_{1}$ and $\mathrm{d}_{2}$ at the time instants $t_{1}$ and $t_{2}\left(t_{2}>t_{1}\right)$ respectively, then, during the duration $\mathrm{T}=\mathrm{t}_{2}-\mathrm{t}_{1}$ of the time interval $\left(\mathrm{t}_{1}, \mathrm{t}_{2}\right)$, the UE client has an average relative velocity of

$\bar{v}_{1,2}=\left(\mathrm{d}_{2}-\mathrm{d}_{1}\right) / \mathrm{T}=\left|\bar{v}_{1,2}\right| \operatorname{sgn}\left(\bar{v}_{1,2}\right)$

with respect to the hotspot, during the time interval $\mathrm{T}=\mathrm{t}_{2}-\mathrm{t}_{1}$.

In Eq. 1 [20], the magnitude $\left|\bar{v}_{1,2}\right|$ of $\bar{v}_{1,2}$ indicates how fast the UE client is approaching towards or receding from the hotspot, i.e., $\left|\bar{v}_{1,2}\right|$ indicates the magnitude of the speed of progression or regression of the UE client, relative to the hotspot. On the other hand, sgn $\left|\bar{v}_{1,2}\right|$ signifies whether the client is moving towards [if $\operatorname{sgn}\left|\bar{v}_{1,2}\right|<0$ ] or away from [sgn| $\left.\bar{v}_{1,2} \mid>0\right]$ the hotspot, i.e., whether the motion of the UE client, relative to the hotspot, is progressive or regressive. Obviously, if the motion of the UE client relative to a particular hotspot is the maximum or the highest progressive among the motions to all the hotspots, it may be chosen for connection by the UE client if the proximity is satisfactory. Thus based on the self-estimation of periodic distances calculated from few periodic samples of the RSS values from each hotspot, the UE client can perform the look ahead.

Thus the values $\left\{\Delta_{\mathrm{i}-1, \mathrm{i}}\right\}=\left\{\mathrm{d}_{\mathrm{i}}-\mathrm{d}_{\mathrm{i}-1}\right\}$ of the successive "differences in consecutive distances" (DiCD) of the UE client from a hotspot themselves represent the average velocity (after scaling by the factor $1 / \mathrm{T}$ ) of the UE client, relative to the hotspot, during the respective equal time intervals $\left(t_{1}, t_{2}\right),\left(t_{2}, t_{3}\right)$ and so on [20]. Accordingly, each individual DiCD may, generally speaking, be given by the following vector:

$\Delta_{\mathrm{i}-1, \mathrm{i}}=\mathrm{d}_{\mathrm{i}}-\mathrm{d}_{\mathrm{i}-1}=\left|\mathrm{d}_{\mathrm{i}}-\mathrm{d}_{\mathrm{i}-1}\right| \operatorname{sgn}\left(\mathrm{d}_{\mathrm{i}}-\mathrm{d}_{\mathrm{i}-1}\right)$

The UE client assigns the magnitude-cum-DoM based scores ( $\left.\mathrm{S}_{\mathrm{MDoM}}\right)$ to each hotspot depending on the magnitude $\left|\bar{v}_{\mathrm{i}-1, \mathrm{i}}\right|$ and the DoM sgn $\left(\bar{v}_{\mathrm{i}-1, \mathrm{i}}\right)$ of the average relative velocity of the UE client with respect to the concerned hotspot during the time interval $\left(\mathrm{t}_{\mathrm{i}}-\mathrm{t}_{\mathrm{i}-1}\right)$. The scoring is based on the DiCDs. Referring to Equation 2, for every $\left(\mathrm{d}_{\mathrm{i}}-\mathrm{d}_{\mathrm{i}-1}\right)$ calculated for each hotspot, a UE client assigns the $\mathrm{S}_{\mathrm{MDoM}}$ in the following manner at the time instants $\left\{\mathrm{t}_{\mathrm{i}}\right\}, \mathrm{i}=2,3,4, \ldots$

- For $\Delta_{l, 2}$, if $\operatorname{sgn}\left(d_{2}-d_{l}\right)<0$ (i.e., if the UE client's DoM with respect to the hotspot is progressive), then the magnitude $\left|d_{2}-d_{1}\right|$ is weighted, i.e., multiplied by +1 ; else, if $\operatorname{sgn}\left(d_{2}-d_{1}\right)>0$, then the magnitude $\left|d_{2}-d_{1}\right|$ is weighted by -1 . Similarly,

- For $\Delta_{2,3}$, if $\operatorname{sgn}\left(d_{3}-d_{2}\right)<0$, then the magnitude $\left|d_{3}-d_{2}\right|$ is weighted by +2 ; else, if $\operatorname{sgn}\left(d_{3}-d_{2}\right)>0$, then the magnitude $\left|d_{3}-d_{2}\right|$ is weighted by -2 .

- For $\Delta_{3,4}$, if $\operatorname{sgn}\left(d_{4}-d_{3}\right)<0$, then the magnitude $\left|d_{4}-d_{3}\right|$ is weighted by +3 ; else, if $\operatorname{sgn}\left(d_{4}-d_{3}\right)>0$, then the magnitude $\left|d_{4}-d_{3}\right|$ is weighted by -3 , and so on.
The above gradual increase in the weighting factor is intended to underscore the greater importance of the more recent magnitudes of the velocity compared to the earlier ones, i.e., to emphasize the latest status of the relative motion. This would ensure that the hotspots towards which the UE client is approaching the fastest till the last moment will most likely be chosen for connection. The $\mathrm{S}_{\mathrm{MDoM}}$ will be computed by summing the scores assigned to $\Delta_{1,2}, \Delta_{2,3}, \Delta_{3,4}$, etc. The overall NoM-based $\mathrm{S}_{\mathrm{NoM}}$ will be computed by summing up $\mathrm{S}_{\mathrm{PRO}}$ and $\mathrm{S}_{\mathrm{MDoM}}$ after assigning them appropriately chosen weights $\mathrm{W}_{\text {PRO }}$ and $\mathrm{W}_{\text {MDoM }}$. The weights should give the proper relative importance to both proximity and the MDoM of the UE client relative to the chosen hotspot.

\section{B. Assignment of Connection-based Score $\left(S_{\mathrm{CON}}\right)$}

How a hotspot can estimate the number of new connections it can serve at any time will be discussed under subsection $\mathrm{C}$. We will assume that the highest number of connections that a hotspot can offer, i.e., invite at any time is $\mathrm{N}$. This maximum number $\mathrm{N}$ is chosen by a hotspot if it has enough LoE to sustain $\mathrm{N}$ connections (assuming each connection lasts a chosen average time duration), after leaving aside the amount of energy needed for its own use. However, if the hotspot is presently serving M UE clients, it can invite only $\mathrm{P}=\mathrm{N}-\mathrm{M}$ connections. The connection-based score $\mathrm{S}_{\mathrm{CON}}=\mathrm{P}$ will be assigned to a particular hotspot by an UE client if that hotspot is advertising or offering $\mathrm{P}$ connections.

\section{Hotspot Estimating the Number of New Connections it can Serve}

An UE hotspot can assess the number of new connections it can serve before its battery energy drops to the threshold value reserved for its own use. The number of connections it can invite at any time depends on its LoE and the number of its existing connections at that time. We assume that the smartphone hotspot's battery has an initial stored energy E. Out of that total energy, an amount of energy, $e_{t}$, to be called the "threshold energy", is always kept reserved for meeting the hotspot's own need of energy (i.e., to keep running and to make calls or open internet connection) for a chosen period of time. The remaining energy $\left(E-e_{t}\right)$ may be utilized by the hotspot for powering the invited connections from UE clients. Generally, a smartphone operating in a WiFi tethering mode is able to serve 5-8 other WiFi-enabled devices (assuming one connection per device). For this proposal, we assume that a hotspot can only service a maximum of 5 connections (one connection per UE client) and a minimum of 0 connections at any time. The actual number of invited connections at any time will depend on the LoE of the battery at that time, the threshold energy and the number of connections that already exists at the hotspot at that time.

Now the LoE of the hotspot's battery at any time may be estimated as follows. We assume that, using some appropriate instrumentation, we can periodically (say, every $T$ seconds) monitor the instantaneous power $\mathrm{P}$ that is being delivered by the battery. So, assuming the instantaneous power of the hotspot's battery at the times T sec, 2 T sec, 3 T sec, etc. (after 
the battery has been fully charged), to be $\mathrm{P}_{1}, \mathrm{P}_{2}, \mathrm{P}_{3}$, etc., we can measure the LoE of the battery at $T \mathrm{sec}, 2 \mathrm{Tsec}, 3 \mathrm{Tsec}$, etc., as E- $\mathrm{P}_{1} \mathrm{~T}$, E- $\left(\mathrm{P}_{1}+\mathrm{P}_{2}\right) \mathrm{T}$, E- $\left(\mathrm{P}_{1}+\mathrm{P}_{2}+\mathrm{P}_{3}\right)$ T, etc. Let us denote the LoE of the battery at any arbitrary time $t$ as e. Then it is obvious that the number of connections that can be advertised by the hotspot at any arbitrary time will depend on $\mathrm{e}$ in the following manner.

- If $0<e \leq e_{t}$, then the number of advertised connections is 0

- If $e_{t}<e \leq(1 / 5)\left(E-e_{t}\right)$, then the number of advertised connections is 1

- If $(1 / 5)\left(E-e_{t}\right)<e \leq(2 / 5)\left(E-e_{t}\right)$, then the number of advertised connections is 2

- If $(2 / 5)\left(E-e_{t}\right)<e \leq(3 / 5)\left(E-e_{t}\right)$, then the number of advertised connections is 3

- If $(3 / 5)\left(E-e_{t}\right)<e \leq(4 / 5)\left(E-e_{t}\right)$, then the number of advertised connections is 4

- If $(4 / 5)\left(E-e_{t}\right)<e \leq\left(E-e_{t}\right)$, then the number of advertised connections is 5

Thus the number of connections that may be advertised or invited by the hotspot at any time is the number of connections that can be sustained (leaving aside the threshold energy $e_{t}$ ) less the number of existing connections. Each UE hotspot then advertises that number along with other relevant association credentials for the potential UE clients. UE clients then assign the connection scores $\mathrm{S}_{\mathrm{CON}}$ to each hotspot. Obviously, the hotspot with the highest $\mathrm{S}_{\mathrm{CON}}$ gets the maximum score, which is proportional to its number of invited connections.

\section{UE Client Assigning the Overall WAS}

The UE client, finally, calculates the overall WAS by combining $\mathrm{S}_{\mathrm{NoM}}$ with $\mathrm{S}_{\mathrm{CON}}$ after assigning appropriate weights to each. It then chooses the hotspot with the highest overall WAS as the most suitable one to connect to. For calculating the overall WAS, weights of the two parameters, $W_{\mathrm{NoM}}$ and $\mathrm{W}_{\mathrm{CON}}$, are assigned to 0.4 and 0.6 , respectively. We have assigned more weightage to $\mathrm{S}_{\mathrm{CON}}$ as the quality of a UE client's communication is very important in a natural disaster situation and that may suffer if connected to a hotspot which has little LoE for sustaining new communication. Thus, even if a hotspot has received a very good NoM score, an UE client should not choose it if it's $\mathrm{S}_{\mathrm{CON}}$ is low.

\section{An ILLUSTRATIVE EXAMPLE OF SCORE ASSIGNMENT}

We discuss here the proposed mechanism with the help of an illustrative example. In Figure 1, we consider a disaster affected environment having a congested eNB with little LoE. $\mathrm{A}, \mathrm{B}, \mathrm{C}$ and $\mathrm{D}$ are the temporary hotspots, under the coverage of the eNB, providing internet connectivity and communication to other WiFi-enabled UE clients (indicated as UE in the diagram) using WiFi tethering. As mentioned in Section IV.C, each temporary hotspot can serve a maximum of five connections (i.e., five UE clients). The existing number of connections per hotspot is shown in the figure. A new WiFienabled UE client approaching the coverage area of the eNB

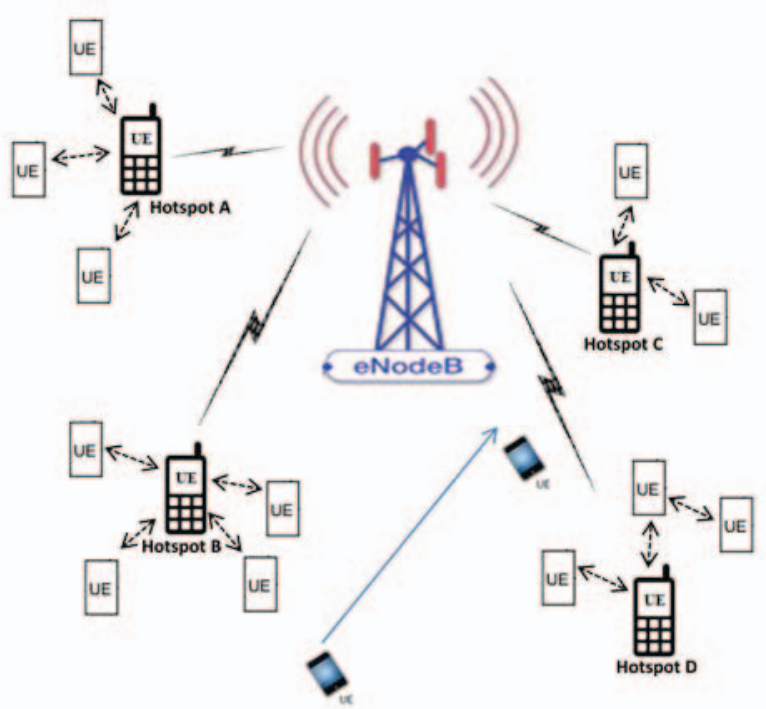

Fig. 1: UE client's selection of the most suitable hotspot

and moving in the direction of the arrow, has to select the most suitable hotspot, out of the four, to connect to.

We assume that the UE client performs four scanning cycles of the environment at regular intervals and records four RSS values per hotspot. The client records sixteen RSS values in total and therefore, calculates sixteen different distance values (four per hotspot). Let's say, the distance values are

$$
\begin{aligned}
& \mathrm{d}_{\mathrm{A} 1}=50, \mathrm{~d}_{\mathrm{B} 1}=12, \mathrm{~d}_{\mathrm{C} 1}=30, \mathrm{~d}_{\mathrm{D} 1}=40 \\
& \mathrm{~d}_{\mathrm{A} 2}=60, \mathrm{~d}_{\mathrm{B} 2}=27, \mathrm{~d}_{\mathrm{C} 2}=22, \mathrm{~d}_{\mathrm{D} 2}=30 \\
& \mathrm{~d}_{\mathrm{A} 3}=65, \mathrm{~d}_{\mathrm{B} 3}=40, \mathrm{~d}_{\mathrm{C} 3}=15, \mathrm{~d}_{\mathrm{D} 3}=25 \\
& \mathrm{~d}_{\mathrm{A} 4}=72, \mathrm{~d}_{\mathrm{B} 4}=50, \mathrm{~d}_{\mathrm{C} 4}=8, \mathrm{~d}_{\mathrm{D} 4}=18
\end{aligned}
$$

where, $\mathrm{d}_{\mathrm{A} 1}, \mathrm{~d}_{\mathrm{B} 1}, \mathrm{~d}_{\mathrm{C} 1}$ and $\mathrm{d}_{\mathrm{D} 1}$ are the calculated distances for hotspots $\mathrm{A}, \mathrm{B}, \mathrm{C}$ and $\mathrm{D}$, respectively, from the RSS values recorded at the first scanning cycle. Similarly, the other distances are calculated from RSS values recorded during scanning cycles two, three and four. The value of the maximum distance to any hotspot computed over all the scanning cycles is 72 .

As explained in Section IV.A, the proximity-based score ( $\mathrm{S}$ PRO), assigned by the UE client to individual hotspots, is simply the difference between 72 (i.e., the maximum distance value) and the most recently computed distance to a particular hotspot, i.e., the distance computed from RSS values recorded during the last (fourth) scanning cycle. Thus, SPRo for hotspots $\mathrm{A}, \mathrm{B}, \mathrm{C}$ and $\mathrm{D}$, respectively, are $0,22,64$ and 54 . Next, the UE client assigns the MDoM scores $\left(\mathrm{S}_{\mathrm{MDoM}}\right)$ to individual hotspots in the following way (refer to Section IV.A). The scoring is based on each DiCD and depends on the magnitude and the DoM (i.e., whether progressive or regressive) of the average relative velocity of the UE client with respect to each hotspot during a certain time interval. Referring to Equation 2, DiCDs for hotspot A are

$$
\Delta_{1,2}=(60-50)=+10 ; \Delta_{2,3}=+5 ; \text { and } \Delta_{3,4}=+7
$$


So, the calculated magnitudes for three DiCDs are 10, 5 and 7 and the DoM sgn $\left(\bar{v}_{\mathrm{i}-1, \mathrm{i}}\right)$ is positive for all (which implies a regressive DoM of the UE client with respect to the hostpot). Thus, the scores assigned to $\Delta_{1,2}, \Delta_{2,3}$ and $\Delta_{3,4}$ for hotspot $\mathrm{A}$, respectively, are $10 *(-1)=-10,5 *(-2)=-10$ and $7 *(-3)=-21$. Summing these values, the $\mathrm{S}_{\mathrm{MDoM}}$ for hotspot $\mathrm{A}$ is calculated as -41 . Similarly, the $S_{\mathrm{MDoM}}$ values calculated for hotspots $B$, $\mathrm{C}$ and $\mathrm{D}$ are $-71,+43$ and +41 , respectively. The $\mathrm{S}_{\mathrm{NoM}}$ for the individual hotspots is then computed based on the weighted sum of the $\mathrm{S}_{\mathrm{PRO}}$ and $\mathrm{S}_{\mathrm{MDoM}}$. For this example, we have assigned a weight of 0.5 to both the parameters. $\mathrm{S}_{\mathrm{NoM}}$ for hotspot $\mathrm{A}$ is thus calculated as

$$
S_{P R O}\left(\text { hotspot }_{A}\right) * W_{P R O}+S_{M D o M}\left(\text { hotspot }_{A}\right) * W_{M D o M}=-20.5
$$

Similarly, the $\mathrm{S}_{\mathrm{NoM}}$ for hotspots $\mathrm{B}, \mathrm{C}$ and $\mathrm{D}$ are, respectively, calculated as $-24.5,+53.5$ and +47.5 .

The UE client then assigns the $\mathrm{S}_{\mathrm{CON}}$ based on the number of new connections an individual hotspot is able to serve before its battery energy drops to a certain threshold. As explained in Section IV.B, if a hotspot is presently serving M UE clients, it can invite only $\mathrm{P}=\mathrm{N}-\mathrm{M}$ connections, where $\mathrm{N}=5$ as per our assumption. Thus, referring to Figure 1, the number of connections that hotspots A, B, C and D can further invite is 2, 1, 3 and 2, respectively. This implies, as well, the $\mathrm{S}_{\mathrm{CON}}$ for the individual hotspots. Finally, the UE client calculates the overall WAS for each hotspot by combining the $\mathrm{S}_{\mathrm{NoM}}$ and $\mathrm{S}_{\mathrm{CON}}$ for each of them and assigning the weights of 0.4 and 0.6 (assumed for this example) to $\mathrm{W}_{\mathrm{NoM}}$ and $\mathrm{W}_{\mathrm{CON}}$. Thus the overall WAS for hotspot A is calculated as

$$
S_{\mathrm{NoM}}\left(\text { hotspot }_{A}\right) * W_{\mathrm{NoM}}+S_{\mathrm{CON}}\left(\text { hotspot }_{A}\right) * W_{\mathrm{CON}}=-7
$$

Similarly, the overall WAS for hotspots B, C and D are calculated as $-9.2,+23.2$ and +20.2 , respectively. The UE client chooses hotspot $\mathrm{C}$, with the highest overall WAS, as the most suitable hotspot to connect to.

\section{CONCLUSIONS}

We proposed a smartphone-based post-disaster management mechanism in natural-disaster affected areas using WiFi tethering. Smartphones belonging to users stuck within the coverage areas of disaster-affected congested eNBs running under low battery backups, may turn themselves into temporary mobile hotspots and provide internet connectivity to other WiFi-enabled clients. The novelties of this scheme are the following: (a) the smartphone hotspots can self-assess the number of new UE client connections they can serve based on their LoE of the battery, and (b) new UE clients can self-select the most suitable temporary hotspots to connect to based on their proximity as well as magnitude and DoM with respect to individual hotspots. The scheme aims to relieve the congested eNBs from getting more congested with the addition of new traffic, and die down soon. Moreover, the new UE clients can sustain important communication for at least some time through the temporary mobile hotspots.

\section{REFERENCES}

[1] T. Fenwick, "Emergency Telephone Call Services and the February 2011 ChCh Earthquake: A Review for the Ministry of Economic Development", August 2011

[2] Y. Nemoto, K. Hamaguchi, "Resilient ICT Research Based on Lessons Learned from the Great East Japan Earthquake" in IEEE Communications Magazine, Vol. 52, Issues 3, pp. 38-43, March 2014

[3] "Maintaining Communications Capabilities during Major Natural Disasters and Other Emergency Situations", Study Group Report, Dec 2011

[4] M. Raj, K. Kant, S. K. Das, "E-Darwin: Energy Aware Disaster Recovery Network using WiFi Tethering", in Proc. of $23^{\text {rd }}$ International Conference on Computer Communication and Networks (ICCCN), Shaghai, China, 4-7 Aug 2014, pp. 1-8

[5] S. Tomoyoshi, M. Yasuhiro, I. Atsushi, "Efforts to Solve the Congestion Problems of Mobile Communications Services during Major Natural Disasters", NEC Technical Journal, Vol. 7, No. 3, pp. 134-139, March 2013

[6] S. K. Ray et. al., "An Energy Aware Mobile-Controlled Handover Method for Natural Disaster Situations", in Proc. of $10^{\text {th }}$ Australasian Telecommunication Networks and Applications Conference (ATNAC), Christchurch, New Zealand, 20-22 Nov 2013, pp. 130-135

[7] J. Blanchard, "Smart Energy Solutions Using Fuel Cells", in IEEE 33 $3^{\text {rd }}$ International Telecommunications Energy Conference (INTELEC), Amsterdam, Netherlands, 9-13 October 2011, pp. 1-8

[8] "Hydrogen Powered Base Stations for Coverage in Disasters and Blackouts", in URL: http://www.gsma.com/publicpolicy/ hydrogenpowered-base-stations-for-coverage-in-disasters-and-blackouts

[9] Y. Shibata, N. Uchida, N. Shiratori, “Analysis of and Proposal for a Disaster Information Network from Experience of the Great East Japan Earthquake", IEEE Communications Magazine, Vol. 52, Issue 3, pp. 4458, March 2014

[10] Q. T. Minh, K. Nguyen, C. Borcea, S. Yamada, "On-the-Fly Establishment of Multihop Wireless Access Networks for Disaster Recovery”, IEEE Communications Magazine, Vol. 52, Issue 10, pp. 6066, October 2014

[11] D. Iland, E. M. Belding, "EmergeNet: Robust, Rapidly Deployable Cellular Networks", IEEE Communications Magazine, Vol. 52, Issue 12, pp. 74-80, December 2014

[12] K. Mase, "Communications Supported by Ad Hoc Networks in Disasters", Journal of the IEICE, vol. 89, No. 9, pp. 796-800, 2006.

[13] T. Fujiwara and T. Watanabe, "An Ad Hoc Networking Scheme in Hybrid Networks for Emergency Communications", Ad Hoc Networks, vol. 3, No. 5, pp. 607-620, 2005.

[14] M. Muraai, T. Furuya, T. Imai, S. Kimura, "Application of Server Vurtualization Technology to Communication Services", Fujitsu Sci. Tech. Journal, Vol. 49, No. 3, pp. 286-291, July 2013

[15] S. K. Ray, N. I. Sarkar, D. Deka, S. Ray, "LTE-Advanced based Handover Mechanism for Natural Disaster Situations", in Proc. of $29^{\text {th }}$ International Conference on Information Networking (ICOIN 2015), Siem Reap, Cambodia, 12-14 Jan 2015

[16] “The LTE-Network Architecture: A Comprehensive Tutorial”, Strategic White Paper, Alcatel Lucent, 2009

[17] L. Yu et. al., "A Novel Optimizing Mechanism of Load Balancing in LTE Network", Advanced Materials Research, Vol. 756-759, pp. 32933297, Sept 2013

[18] "IEEE P802.16q System Requirement Document", Draft Working Document, January 2013

[19] S. K. Ray, J. Kumar, S. Sen, and J. Nath, "Modified distance vector routing scheme for a MANET," in Proc. of The National Conference on Communications (NCC), Kanpur, India, January 2007, pp. 197-202.

[20] S. K. Ray et. al., "Self-Tracking Mobile Station Controls Its Fast Handover in Mobile WiMAX", in Proc. of IEEE Wireless Communications and Networking Conference (WCNC), Sydney, Australia, 18-21 April 2010, pp. 1-6 